\title{
ON THE SECOND DERIVATIVES OF AN EXTREMAL-INTEGRAL WITH AN APPLICATION TO A PROBLEM WITH
}

\author{
VARIABLE END POINTS*
}

BY

\author{
ARNOLD DRESDEN
}

\section{INTRODUCTION}

In a paper published in volume 9 of these $\operatorname{Trans}$ a c t i o n s, formulæ were obtained for the second derivatives of the extremal-integral arising in the problem of minimizing or maximizing the integral $\int F\left(x, y, x^{\prime}, y^{\prime}\right) d t$. In these formulæ the derivatives were expressed in terms of particular solutions of the Weierstrass form of Jacobi's differential equation. $\dagger$ They were used in the same paper to obtain further necessary conditions for a minimum of the above mentioned integral, when one or both endpoints were variable along a curve, $\ddagger$ or when curves with discontinuous slopes were admitted as solutions of the problem. It is the author's aim to use the same general method in analogous problems involving integrals of a more general type, and also in the cases in which the unknown functions are further conditioned by differential and algebraic equations. As a first step in this direction, we obtain in the present paper the second derivatives of the extremal-integral arising in the theory of the integral

$$
\int f\left(x, y_{1}, \cdots, y_{n} ; y_{1}^{\prime}, \cdots, y_{n}^{\prime}\right) d x \quad\left(y_{i}^{\prime}=d y_{i} / d x\right),
$$

and express them in terms of particular solutions of the differential equations which correspond to Jacobi's equations for the simpler problem. The formulæ for these derivatives are then used for finding further necessary conditions to be satisfied by a curve $C$ in the space of $n+1$ dimensions which is to furnish a

* The contents of sections 1 and 2 of this paper are contained in the paper presented to the Society at the Chicago meeting of December, 1914, an abstract of which appeared in the Proceedings of the National Academy of Sciences for March, 1914.

† Compare Bolza, Vorlesungen über Variationsrechnung, p. 306-313 (this book will be referred to in the sequel by the author's name only), and Dresden, these Transactions, vol. 9 (1908), pp. 467 and 472.

$\ddagger$ The conditions here referred to had been found before by Bliss, these $\mathrm{T}$ r a $\mathrm{n}$ s a c t i o $\mathrm{ns}$, vol. 3 (1902), p. 136, and $M$ athematis che Annalen, vol. 58 (1903), p. 70. 
minimum for the integral (1), when the initial point of $C$ is allowed to move along a fixed curve $L$.*

A number of familiar facts concerning the integral (1) which will be needed in the development of our problem have been collected in section 1 . In section 2 the formulæ for the second derivatives of the extremal integral are obtained, and in section 3 these formulæ are applied to the problem with variable initial point.

Concerning the function $f$, which appears in the integral (1), we assume that it is of class $C^{\prime \prime \prime}$ with respect to all of its arguments; the functions $y(x)$ which are admitted as solutions of our problem must be of class $C^{\prime \prime}$. We shall moreover use the following abbreviated notations:

$$
f_{0}=\partial f / \partial x, \quad f_{i}=\partial f / \partial y_{i}, \quad f_{n+i}=\partial f / \partial y_{i}^{\prime},
$$

and similar notations will be used for the second derivatives. A point in $n+1$ dimensional space of coördinates $x, y_{1}, \cdots, y_{n}$ will be designated by $(x ; y)$, and similar notations will be used for sets of numbers which can be interpreted as coördinates of points in spaces of higher dimensionality. Finally the notation $f^{(i)}$ will be used to indicate that the function $f\left(x ; y ; y^{\prime}\right)$ is to be taken with the arguments $\left(x_{i} ; y_{i} ; y_{i}^{\prime}\right)$.

\section{Preliminary developments}

Any set of functions $y_{1}(x), \cdots, y_{n}(x)$ which is to furnish a minimum or a maximum for the integral (1) must satisfy the system of differential equations

$$
\frac{\partial f}{\partial y_{i}}-\frac{d}{d x} \frac{\partial f}{\partial y_{i}^{\prime}}=0 \quad(i=1, \cdots, n),
$$

known as Euler's differential equations. $\dagger$ Such a set of functions defines a curve in the space of $n+1$ dimensions, called an extremal for the problem; we shall use repeatedly geometric terminology consistent with this interpretation of the set of functions $y_{i}(x)$. From the general existence theorems for differential equations, $\ddagger$ we know that the general solution of the system of differential equations (2) is of the form

$$
y_{i}=g_{i}\left(x ; \alpha_{1}, \cdots, \alpha_{n} ; \beta_{1}, \cdots, \beta_{n}\right) \quad(i=1, \cdots, n),
$$

the functions $g_{i}$ being of class $C^{\prime \prime}$ with respect to all of their arguments and $\alpha_{1}, \cdots, \beta_{n}$ being $2 n$ arbitrary constants.

* For the case $n=2$, this problem has been treated by Miss M. B. White in her paper, The dependence of focal points upon curvature for problems of the calculus of variations in space, these Trans a ction s, vol. 13 (1912), p. 175.

† See Bolza, p. 542.

† Ibid., pp. 175-179. 
We suppose now that we have found an extremal passing through the two points $A\left(a_{0} ; a\right)$ and $B\left(b_{0} ; b\right)$ and satisfying the conditions of Clebsch and Mayer.* The second variation of the integral (1) can then be put into the form

$$
\delta^{2} I=\frac{\epsilon^{2}}{2} \int_{a_{0}}^{b_{0}} \sum_{i k}\left(P_{i k} \eta_{i} \eta_{k}+2 Q_{i k} \eta_{i} \eta_{k}^{\prime}+R_{i k} \eta_{i}^{\prime} \eta_{k}^{\prime}\right) d x,
$$

where $\eta_{1}, \cdots, \eta_{n}$ are arbitrary functions of $x$ of class $C^{\prime}$ vanishing for $x=a_{0}$ and for $x=b_{0}$, and where $P_{i k}=f_{i k}, Q_{i k}=f_{i, n+k}$ and $R_{i k}=f_{n+i, n+k}$, these functions being taken along the given extremal. If the integrand which ap. pears in this formula for the second variation be denoted by $2 \Omega$, and if we write

$$
\psi_{i}(\eta)=\frac{\partial \Omega}{\partial \eta_{i}}-\frac{d}{d x} \frac{\partial \Omega}{\partial \eta_{i}^{\prime}},
$$

we obtain, by means of Euler's theorem on homogeneous functions and by the familiar integration by parts, the following result:

$$
\delta^{2} I=\frac{\epsilon^{2}}{2} \int_{a_{0}}^{b_{0}} \sum_{i} \eta_{i} \psi_{i} d x .
$$

The system of equations

$$
\psi_{i}(\eta)=0, \quad(i=1, \cdots, n),
$$

is a system of linear differential equations of the second order in the unknown functions $\eta_{i}$; it is a self-adjoint system $\dagger$ and is called, after Von Escherich, the accessory system of differential equations; $\ddagger$ we observe moreover that it is the direct analogue of Jacobi's equation for the simplest problem of the calsulus of variations. It follows further $\S$ that a fundamental system of $2 n$ solutions of the equations (4) may be obtained by differentiating the furctions $g_{i}$, which appear in the equations (3), with respect to each of the parameters $\alpha_{1}, \cdots, \beta_{n}$ and then replacing these by $\alpha_{10}, \cdots, \beta_{n 0}$, the latter being the values which furnish the given extremal if put in place of $\alpha_{1}, \cdots, \beta_{n}$ in equations (3). We obtain in this way the following $2 n$ systems of solutions of equations (4):

$$
\partial g_{1} / \partial \alpha_{i}, \cdots, \partial g_{n} / \partial \alpha_{i} ; \quad \partial g_{1} / \partial \beta_{i}, \cdots, \partial g_{n} / \partial \beta_{i}, \quad(i=1, \cdots, n),
$$

the arguments of these functions being $x ; \alpha_{10}, \cdots, \beta_{n 0}$.

We consider now two points $P_{1}\left(x_{1} ; y_{1}\right)$ and $P_{2}\left(x_{2} ; y_{2}\right)$ in the neighborhood of $A\left(a_{0} ; a\right)$ and $B\left(b_{0} ; b\right)$, respectively. Since the extremal $A B$ satisfies the Mayer condition, we can conclude that it is possible to construct an

* Bolza, p. 608 and p. 619.

$\dagger$ Hadamard, Leçons sur le Calcul des Variations, p. 319.

$\ddagger$ Bolza, p. 622; further references are given there.

§ Bolza, p. 623 and Hadamard, loc. cit., pp. 336-339. 
unique extremal through the points $P_{1}$ and $P_{2}$, i. e., that it is possible to solve the equations

$$
y_{1 i}=g_{i}\left(x_{1} ; \alpha_{1}, \cdots, \beta_{n}\right), \quad y_{2 i}=g_{i}\left(x_{2} ; \alpha_{1}, \cdots, \beta_{n}\right)
$$

uniquely for $\alpha_{i}$ and $\beta_{i}$ in terms of $x_{1}, x_{2}, y_{1 j}$ and $y_{2 j} .{ }^{*}$ We denote the solutions of these equations by:

$$
\alpha_{i}=A_{i}\left(x_{1}, x_{2} ; y_{11}, \cdots, y_{2 n}\right), \quad \beta_{i}=B_{i}\left(x_{1}, x_{2} ; y_{11}, \cdots, y_{2 n}\right) .
$$

Substitution of equations (7) in equations (3) furnishes the following analytical representation for the extremal through the points $P_{1}$ and $P_{2}$ :

$$
\begin{aligned}
y_{i}=g_{i}\left(x ; A_{1}\left(x_{1}, \cdots, y_{2 n}\right), \cdots, B_{n}\left(x_{1}, \cdots, y_{2 n}\right)\right) \\
\quad \equiv Y_{i}\left(x ; x_{1}, x_{2} ; y_{11}, \cdots, y_{2 n}\right) .
\end{aligned}
$$

Upon substituting from equations (7) in equations (6), we see that the functions $Y_{i}$, which are defined by equations (8), satisfy the following initial conditions:

(9) $\quad Y_{i}\left(x_{1} ; x_{1}, x_{2} ; y_{11}, \cdots, y_{2 n}\right)=y_{1 i}, \quad Y_{i}\left(x_{2} ; x_{1}, x_{2}, y_{11}, \cdots, y_{2 n}\right)=y_{2 i}$.

Differentiation of these equations with respect to $x_{1}, x_{2}, y_{11}, \cdots, y_{2 n}$ furnishes furthermore the following relations, which will be of use in the sequel:

$$
\begin{array}{lll}
\partial Y_{i} /\left.\partial x_{1}\right|_{1}+Y_{i}^{\prime}\left(x_{1}\right)=0, & \partial Y_{i} /\left.\partial x_{2}\right|_{2}+Y_{i}^{\prime}\left(x_{2}\right)=0, \\
\partial Y_{i} /\left.\partial y_{2 j}\right|_{1}=0, & \partial Y_{i} /\left.\partial x_{2}\right|_{1}=0, & \partial Y_{i} /\left.\partial y_{1 j}\right|_{1}=\delta_{i j}, \\
\partial Y_{i} /\left.\partial x_{1}\right|_{2}=0, & \partial Y_{i} /\left.\partial y_{1 j}\right|_{2}=0, & \partial Y_{i} /\left.\partial y_{2 j}\right|_{2}=\delta_{i j},
\end{array}
$$

or, expressed in different form,

$$
\begin{aligned}
& g_{i}^{\prime}\left(x_{1}\right)+\left.\sum_{j}\left\{\frac{\partial g_{i}}{\partial \alpha_{j}} \frac{\partial A_{j}}{\partial x_{1}}+\frac{\partial g_{i}}{\partial \beta_{j}} \frac{\partial B_{j}}{\partial x_{1}}\right\}\right|_{1}=0 \\
& g_{i}^{\prime}\left(x_{2}\right)+\left.\sum_{j}\left\{\frac{\partial g_{i}}{\partial \alpha_{j}} \frac{\partial A_{j}}{\partial x_{2}}+\frac{\partial g_{i}}{\partial \beta_{j}} \frac{\partial B_{j}}{\partial x_{2}}\right\}\right|_{2}=0
\end{aligned}
$$

(11) $\left.\sum_{j}\left\{\frac{\partial g_{i}}{\partial \alpha_{j}} \frac{\partial A_{j}}{\partial x_{1}}+\frac{\partial g_{i}}{\partial \beta_{j}} \frac{\partial B_{j}}{\partial x_{1}}\right\}\right|_{2}=0,\left.\quad \sum_{j}\left\{\frac{\partial g_{i}}{\partial \alpha_{j}} \frac{\partial A_{j}}{\partial x_{2}}+\frac{\partial g_{i}}{\partial \beta_{j}} \frac{\partial B_{j}}{\partial x_{2}}\right\}\right|_{1}=0$,

$$
\left.\sum_{j}\left\{\frac{\partial g_{i}}{\partial \alpha_{j}} \frac{\partial A_{j}}{\partial y_{1 h}}+\frac{\partial g_{i}}{\partial \beta_{j}} \frac{\partial B_{j}}{\partial y_{1 h}}\right\}\right|_{1}=\delta_{i h},\left.\quad \sum_{j}\left\{\frac{\partial g_{i}}{\partial \alpha_{j}} \frac{\partial A_{j}}{\partial y_{2 h}}+\frac{\partial g_{i}}{\partial \beta_{j}} \frac{\partial B_{j}}{\partial y_{2 h}}\right\}\right|_{1}=0,
$$

\footnotetext{
* Bolza, p. 597.
}

$$
\begin{aligned}
& \left.\sum_{j}\left\{\frac{\partial g_{i}}{\partial \alpha_{j}} \frac{\partial A_{j}}{\partial y_{1 h}}+\frac{\partial g_{i}}{\partial \beta_{j}} \frac{\partial B_{j}}{\partial y_{1 h}}\right\}\right|_{2}=0,\left.\quad \sum_{j}\left\{\frac{\partial g_{i}}{\partial \alpha_{j}} \frac{\partial A_{j}}{\partial y_{2 h}}+\frac{\partial g_{i}}{\partial \beta_{j}} \frac{\partial B_{j}}{\partial y_{2 h}}\right\}\right|_{2}=\delta_{i h}, \\
& (i=1, \cdots, n ; h=1, \cdots, n) .
\end{aligned}
$$


We compute now the integral (1) along the extremal $P_{1} P_{2}$ by substituting the functions $Y_{1}, \cdots, Y_{n}$ and their derivatives with respect to $x$, for $y_{1}, \cdots, y_{n}$ and $y_{1}^{\prime}, \cdots, y_{n}^{\prime}$ respectively in the integral (1). This integral becomes then a function of the $2 n+2$ coördinates of the points $P_{1}$ and $P_{2}$; it is known as the "extremal-integral ":

$$
I\left(x_{1}, x_{2}, y_{11}, \cdots, y_{2 n}\right)=\int_{x_{1}}^{x_{2}} f\left(x ; Y_{1}, \cdots, Y_{n} ; Y_{1}^{\prime}, \cdots, Y_{n}^{\prime}\right) d x .
$$

The first derivatives of this function I with respect to its different arguments were first given by Hamilton, ${ }^{*}$ and we shall use them as a basis for the computation of the second derivatives; we have the following formulæ:

$$
\begin{aligned}
& \partial I / \partial x_{1}=-f\left(x_{1} ; y_{1} ; y_{1}^{\prime}\right)+\sum_{i} f_{n+i}\left(x_{1} ; y_{1} ; y_{1}^{\prime}\right) y_{1 i}^{\prime}, \\
& \partial I / \partial x_{2}=f\left(x_{2} ; y_{2} ; y_{2}^{\prime}\right)-\sum_{i} f_{n+i}\left(x_{2} ; y_{2} ; y_{2}^{\prime}\right) y_{2 i}^{\prime}, \\
& \partial I / \partial y_{1 j}=-f_{n+j}\left(x_{1} ; y_{1} ; y_{1}^{\prime}\right), \quad \partial I / \partial y_{2 j}=f_{n+j}\left(x_{2} ; y_{2} ; y_{2}^{\prime}\right),
\end{aligned}
$$

where $y_{1}, y_{1}^{\prime}$ are abbreviations for the sets $\left(y_{11}, \cdots, y_{1 n}\right)$ and $\left(y_{11}^{\prime}, \cdots, y_{1 n}^{\prime}\right)$ respectively.

\section{2. 'THE SECOND DERIVATIVES OF THE EXTREMAL-INTEGRAL}

For the determination of the second derivatives of the function $I$ it is necessary to know the derivatives with respect to $x_{1}, \cdots, y_{2 n}$ of the quantities $y_{11}^{\prime}, \cdots, y_{1 n}^{\prime}$ and $y_{21}^{\prime}, \cdots, y_{2 n}^{\prime}$ which determine the slope of the extremal $P_{1} P_{2}$, at its end-points. If we denote by $z$ any one of the coördinates of the points $P_{1}$ and $P_{2}$, we obtain immediately, upon differentiation of equations (8), the result,

$$
\frac{\partial Y_{i}}{\partial z}=\sum_{j}\left(\frac{\partial g_{i}}{\partial \alpha_{j}} \frac{\partial A_{j}}{\partial z}+\frac{\partial g_{i}}{\partial \beta_{j}} \frac{\partial B_{j}}{\partial z}\right)
$$

from which the desired derivatives may be obtained by differentiation with respect to $x$. Furthermore equations (11) enable us to determine expressions for $\partial A_{j} / \partial z$ and $\partial B_{j} / \partial z$; for we have there a set of $2 n$ linear non-homogeneous equations for each of the $2 n+2$ sets of $2 n$ unknowns which we get if we let $z$ assume in succession each of its values. These sets of equations all possess finite unique solutions provided Mayer's condition is satisfied along the extremal $P_{1} P_{2}$. That this will indeed be the case, if the points $P_{1}$ and $P_{2}$ are taken sufficiently near to the points $A$ and $B$, respectively, follows from our conditions on the extremal $A B$ and from the continuity of Mayer's determinant: $\dagger$

* Bolza, p. 599.

$\dagger$ Bolza, p. 611. 


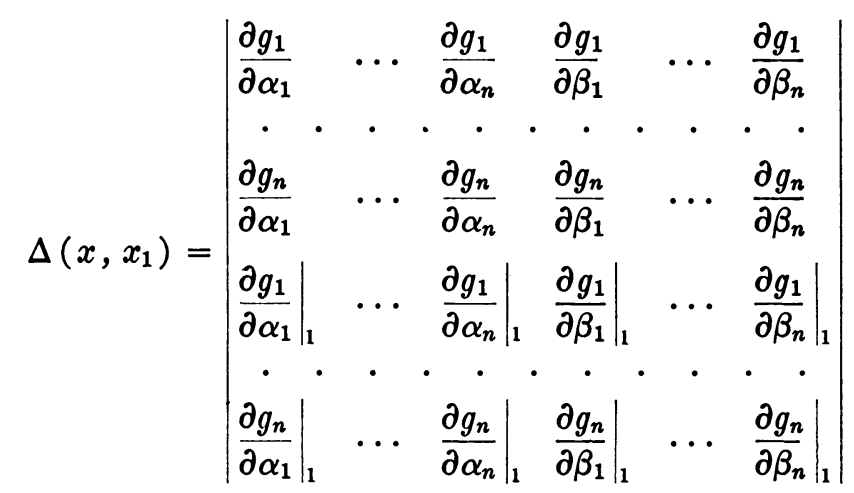

We denote now by $\Delta\left(x, x_{2}\right)$ the determinant obtained from $\Delta\left(x, x_{1}\right)$ by replacing the substitution symbol $\left.\right|_{1}$ in the last $n$ rows by $\left.\right|_{2}$; by $\Delta\left(x_{1}, x_{2}\right)$ the result of replacing the first $n$ rows of $\Delta\left(x, x_{2}\right)$ by the last $n$ rows of $\Delta\left(x, x_{1}\right)$; by $\Delta_{j k}\left(x ; x_{1}, x_{2}\right)$ the result of replacing the $j$ th row of $\Delta\left(x_{1}, x_{2}\right)$ by the $k$ th row of $\Delta\left(x, x_{1}\right)$; and by $\nabla_{j k}\left(x ; x_{1}, x_{2}\right)$ the result of replacing the $(n+j)$ th row of $\Delta\left(x_{1}, x_{2}\right)$ by the $k$ th row of $\Delta\left(x, x_{2}\right)$.

With this notation we can write down the results of solving equations (11) for $\partial A_{j} / \partial z$ and $\partial B_{j} / \partial z$; substitution of them in equations (13) leads to the following formulæ:

$$
\begin{array}{ll}
\frac{\partial Y_{k}}{\partial y_{1 j}}=\frac{\Delta_{j k}\left(x ; x_{1}, x_{2}\right)}{\Delta\left(x_{1}, x_{2}\right)}, & \frac{\partial Y_{k}}{\partial x_{1}}=-\sum_{i} g_{i}^{\prime}\left(x_{1}\right) \frac{\Delta_{i k}\left(x ; x_{1}, x_{2}\right)}{\Delta\left(x_{1}, x_{2}\right)}, \\
\frac{\partial Y_{k}}{\partial y_{2 j}}=\frac{\nabla_{j k}\left(x ; x_{1}, x_{2}\right)}{\Delta\left(x_{1}, x_{2}\right)}, & \frac{\partial Y_{k}}{\partial x_{2}}=-\sum_{i} g_{i}^{\prime}\left(x_{2}\right) \frac{\nabla_{i k}\left(x ; x_{1}, x_{2}\right)}{\Delta\left(x_{1}, x_{2}\right)} .
\end{array}
$$

From these we obtain by direct differentiation the derivatives with respect to $x_{1}, \cdots, y_{2 n}$ of the quantities $y_{11}^{\prime}, \cdots, y_{2 n}^{\prime}$, namely:

$$
\begin{array}{ll}
\frac{\partial^{2} Y_{k}}{\partial x \partial y_{1 j}}=\frac{\Delta_{j k}^{\prime}\left(x ; x_{1}, x_{2}\right)}{\Delta\left(x_{1} ; x_{2}\right)}, & \frac{\partial^{2} Y_{k}}{\partial x \partial x_{1}}=-\sum_{i} g_{i}^{\prime}\left(x_{1}\right) \frac{\Delta_{i k}^{\prime}\left(x ; x_{1}, x_{2}\right)}{\Delta\left(x_{1}, x_{2}\right)}, \\
\frac{\partial^{2} Y_{k}}{\partial x \partial y_{2 j}}=\frac{\nabla_{j k}^{\prime}\left(x ; x_{1}, x_{2}\right)}{\Delta\left(x_{1}, x_{2}\right)}, & \frac{\partial^{2} Y_{k}}{\partial x \partial x_{2}}=-\sum_{i} g_{i}^{\prime}\left(x_{2}\right) \frac{\nabla_{i k}^{\prime}\left(x ; x_{1}, x_{2}\right)}{\Delta\left(x_{1}, x_{2}\right)} .
\end{array}
$$

From the definition of the functions $\Delta_{j k}$ and $\nabla_{j k}$ combined with the properties of the functions (5), it follows that the functions

$$
u_{j k}=\Delta_{j k}\left(x ; x_{1}, x_{2}\right) / \Delta\left(x_{1}, x_{2}\right), \quad v_{j k}=\nabla_{j k}\left(x ; x_{1}, x_{2}\right) / \Delta\left(x_{1}, x_{2}\right)
$$

furnish for every fixed $j$ a solution of the accessory system of differential equations, and that they satisfy the following initial conditions:

$$
u_{j k}\left(x_{1}\right)=\delta_{j k}, \quad u_{j k}\left(x_{2}\right)=0 ; \quad v_{j k}\left(x_{1}\right)=0, \quad v_{j k}\left(x_{2}\right)=\delta_{j k} .
$$

These initial conditions show moreover that each of the systems of functions 
$u_{j k}$ and $v_{j k}$ constitute, in the terminology of Von Escherich, a " conjugate system " of solutions of the accessory system of differential equations.*

We can now express the second derivatives of the extremal-integral in terms of these solutions of the accessory system of differential equations. Differentiation of equations (12) yields, by the use of equations (10) and (15), the following theorem:

Theorem I. The second derivatives of the extremal integral for the integral

$$
\int f\left(x ; y_{1}, \cdots, y_{n} ; y_{1}^{\prime}, \cdots, y_{n}^{\prime}\right) d x
$$

are given by the formula

$$
\begin{aligned}
& I_{1,1}=-f_{0}^{(1)}+\sum_{i} f_{0, n+i}^{(1)} y_{1 i}^{\prime}+\sum_{i j} f_{n+i, n+j}^{(1)} y_{1 i}^{\prime} y_{1 j}^{\prime \prime} \\
& -\sum_{i j k} f_{n+i, n+j}^{(1)} y_{1 i}^{\prime} y_{1 k}^{\prime} u_{k j}^{\prime}\left(x_{1}\right) \\
& I_{2,2}=f_{0}^{(2)}-\sum_{i} f_{0, n+i}^{(2)} y_{2 i}^{\prime}-\sum_{i j} f_{n+i, n+j}^{(2)} y_{2 i}^{\prime} y_{2 j}^{\prime \prime} \\
& +\sum_{i j k} f_{n+i, n+j}^{(2)} y_{2 i}^{\prime} y_{2 k}^{\prime} v_{k j}^{\prime}\left(x_{2}\right) \text {, } \\
& I_{1,2}=-\sum_{i j k} f_{n+i, n+j}^{(1)} y_{1 i}^{\prime} y_{2 k}^{\prime} v_{k j}^{\prime}\left(x_{1}\right)=\sum_{i j k} f_{n+i, n+j}^{(2)} y_{2 i}^{\prime} y_{1 k}^{\prime} u_{k j}^{\prime}\left(x_{2}\right) \text {, } \\
& I_{1,1 i}=-f_{i}^{(1)}+\sum_{j} f_{n+j, i}^{(1)} y_{1 j}^{\prime}+\sum_{j k} f_{n+j, n+k}^{(1)} y_{1 j}^{\prime} u_{i k}^{\prime}\left(x_{1}\right), \\
& =-f_{0, n+i}^{(1)}-\sum_{j} f_{n+i, n+j}^{(1)} y_{1 j}^{\prime \prime}+\sum_{j k} f_{n+i, n+j}^{(1)} y_{1 k}^{\prime} u_{k j}^{\prime}\left(x_{1}\right), \\
& I_{1,2 i}=\sum_{j k} f_{n+j, n+k}^{(1)} y_{1 j}^{\prime} v_{i k}^{\prime}\left(x_{1}\right)=-\sum_{j k} f_{n+i, n+j}^{(2)} y_{1 k}^{\prime} u_{k j}^{\prime}\left(x_{2}\right) \text {, } \\
& I_{2,1 i}=-\sum_{j k} f_{n+j, n+k}^{(2)} y_{2 j}^{\prime} u_{i k}^{\prime}\left(x_{2}\right)=\sum_{j k} f_{n+i, n+j}^{(1)} y_{2 k}^{\prime} v_{k j}^{\prime}\left(x_{1}\right) \text {, } \\
& I_{2,2 i}=f_{i}^{(2)}-\sum_{j} f_{n+j, i}^{(2)} y_{2 j}^{\prime}-\sum_{j k} f_{n+j, n+k}^{(2)} y_{2 j}^{\prime} v_{i k}^{\prime}\left(x_{2}\right) \text {, } \\
& =f_{0, n+i}^{(2)}+\sum_{j} f_{n+i, n+j}^{(2)} y_{2 j}^{\prime \prime}-\sum_{j k} f_{n+i, n+j}^{(2)} y_{2 k}^{\prime} v_{k j}^{\prime}\left(x_{2}\right), \\
& I_{1 i, 1 j}=-\sum_{k} f_{n+i, n+k}^{(1)} u_{j k}^{\prime}\left(x_{1}\right)-f_{n+i, j}^{(1)} \\
& =-\sum_{k} f_{n+j, n+k}^{(1)} u_{i k}^{\prime}\left(x_{1}\right)-f_{n+j, i}^{(1)} \text {, } \\
& I_{1 i, 2 j}=-\sum_{k} f_{n+i, n+k}^{(1)} v_{j k}^{\prime}\left(x_{1}\right)=\sum_{k} f_{n+j, n+k}^{(2)} u_{i k}^{\prime}\left(x_{2}\right) \text {, } \\
& I_{2 i, 2 j}=f_{n+i, j}^{(2)}+\sum_{k} f_{n+i, n+k}^{(2)} v_{j k}^{\prime}\left(x_{2}\right)=f_{n+j, i}^{(2)}+\sum_{k} f_{n+j, n+k}^{(2)} v_{i k}^{\prime}\left(x_{2}\right) \text {, }
\end{aligned}
$$

* See Bolza, p. 627; Hadamard, loc. cit., p. 339; also von Escherich, W i e n e r B e r i c h t e, vol. 107 (1898), p. 1222. 
where the functions $u_{i k}$ and $v_{i k}$ are the solutions of the accessory system of differential equations (4) which are characterized by the initial conditions (16). The subscripts of I specify the variables with respect to which the differentiations are made, single subscripts designating the variable $x$, while pairs of subscripts designate the $y$ 's. Thus $I_{2},{ }_{1 i}=\partial^{2} I / \partial x_{2} \partial y_{1 i}$.

From the continuity restrictions imposed upon the function $f$, it follows that the results of differentiating the extremal integral should be independent of the order of differentiation and hence that the two different expressions given for certain of the derivatives in formulæ (17) should be equal to one another. This fact leads to eight relations between the expressions given in theorem I. It is important for certain applications and as a check upon the accuracy of the formulæ themselves, that these relations should be derived directly from the properties of the functions $u_{i k}$ and $v_{i k}$. We shall denote by $(A),(B)$, $(C),(D),(E),(F),(G)$, and $(H)$ the relations resulting from setting equal to one another the two expressions occurring in each of formulæ $\left(17_{3}\right)$ to $\left(17_{10}\right)$ respectively.

In the first place we observe that certain of these relations follow from others; the remaining ones will then be proved to hold as a consequence of the properties of the functions $u_{i k}$ and $v_{i k}$. If in $(G)$ we interchange the indices $i$ and $j$, then multiply through by $y_{1 j}^{\prime}$ and sum with respect to $j$, and finally interchange the indices $j$ and $k$ in the second sum, we obtain $(C)$. If $(G)$ is multiplied through by $y_{2 j}^{\prime}$ and then summed with respect to $j$, and if finally the summation indices in the first sum are interchanged, we obtain $(D)$. Next, we multiply $(D)$ through by $y_{1 i}^{\prime}$, sum with respect to $i$ and replace the indices $i, j$, and $k$ in the second sum by $k, j$, and $i$, respectively; in this way we obtain $(A) . *$

The functions $y_{i}(x)$ which occur in our formulæ are solutions of Euler's differential equations (2). Substituting these functions in the differential equations and carrying out the differentiation in the second member, we obtain the following identity:

$$
f_{0, n+i}+\sum_{k} f_{n+i, k} y_{k}^{\prime}+\sum_{k} f_{n+i, n+k} y_{k}^{\prime \prime}=f_{i} .
$$

We now multiply $(F)$ through by $y_{1 j}^{\prime}$, sum with respect to $j$, interchange the indices $j$ and $k$ on the left side and add to it equations (18) with $x_{1}$ substituted for $x$; in this way we obtain $(B)$. Using $y_{2 j}^{\prime}$ and $x_{2}$ in analogous manner enables us to show that $(H)$ implies $(E)$. It remains therefore to prove $(G)$, $(F)$, and $(H)$.

To prove the last two relations, we remember that $u_{j k}$, and also $v_{j k}$, are conjugate systems of solutions of the differential equations (4); hence we have $\dagger$

* $(A)$ may also be derived from $(C)$.

† Bolza, p. 626; Hadamard, loc. cit., p. 319 


$$
\psi\left(u_{j} ; u_{i}\right)=0,
$$

where $u_{j}$ and $u_{i}$ designate the systems $\left(u_{j 1}, \cdots, u_{j n}\right)$ and $\left(u_{i 1}, \cdots, u_{i n}\right)$, and where

$$
\psi(\eta ; \zeta)=\sum_{k}\left[\eta_{k} \frac{\partial \Omega}{\partial \zeta_{k}^{\prime}}-\zeta_{k} \frac{\partial \Omega}{\partial \eta_{k}^{\prime}}\right] .
$$

The equation $\psi\left(u_{j} ; u_{i}\right)=0$ reduces to

$$
\sum_{k l} u_{j l}\left(Q_{k l} u_{i k}+R_{k l} u_{i k}^{\prime}\right)-\sum_{k l} u_{i l}\left(Q_{k l} u_{j k}+R_{k l} u_{j k}^{\prime}\right)=0 .
$$

If, in this relation we put $x$ equal to $x_{1}$ and make use of the initial conditions to which the functions $u_{j k}$ are subject, we obtain

$$
Q_{i j}+\sum_{k} R_{k j} u_{i k}^{\prime}=Q_{j i}+\sum_{k} R_{k i} u_{j k}^{\prime},
$$

which is equivalent to $(F)$ in virtue of the meaning of the symbols $Q_{i j}$ and $R_{i j}$. The corresponding treatment for the functions $v_{j k}$ will prove the relation $(H)$. Finally we have for any two systems of functions $u_{i k}$ and $v_{j k}$ the relation*

$$
\sum_{k l} v_{j l}\left(Q_{k l} u_{i k}+R_{k l} u_{i k}^{\prime}\right)-\sum_{k l} u_{i l}\left(Q_{k l} v_{j k}+R_{k l} v_{j k}^{\prime}\right)=\text { const. }
$$

If we put $x$ successively equal to $x_{1}$ and to $x_{2}$ and set the two results equal to each other, making use at the same time of the initial conditions on the functions $u_{j k}$ and $v_{j k}$, we obtain $(G)$.

\section{The CASE OF ONE VARIABLE ENDPOINT}

We suppose now that the endpoint $P_{1}$ of our extremal $P_{1} P_{2}$ is allowed to move along a curve $L$ in the space of $n+1$ dimensions, whose equations are

$$
x=\xi(t), \quad y_{i}=\eta_{i}(t), \quad t^{\prime} \leqq t \leqq t^{\prime \prime} .
$$

We suppose that this curve has no singular points, i. e.,

$$
\xi^{\prime 2}(t)+\sum_{i}\left[\eta_{i}^{\prime}(t)\right]^{2} \neq 0, \quad t^{\prime} \leqq t \leqq t^{\prime \prime},
$$

and that it is of class $C^{\prime \prime}$ in the interval $\left(t^{\prime} t^{\prime \prime}\right)$.

We assume furthermore that we have found an extremal $E$

$$
y_{i}=y_{i}(x), \quad x_{1} \leqq x \leqq x_{2},
$$

which satisfies the following conditions:

I. The condition of Clebsch: $\dagger$

* Bolza, p. 626; Hadamard, loc. cit., p. 319.

† Bolza, p. 608. 
At every point of the extremal the quadratic form $\sum_{i k} R_{i k} \zeta_{i} \zeta_{k}$ must be positive, i. e.:

$$
\sum_{i k} R_{i k} \zeta_{i} \zeta_{k}>0, \quad x_{1} \leqq x \leqq x_{2}
$$

II. The condition of Mayer:*

$$
x_{2} \leqq x_{1}^{\prime},
$$

where $x_{1}^{\prime}$ is defined as the root next following upon $x_{1}$ of Mayer's determinant (14). The point $P_{1}^{\prime}$ determined upon $E$ by $x_{1}^{\prime}$ is known as the conjugate point.

III. The value $t_{1}$ of $t$ furnishes on $L$ the point where it intersects the extremal $E$; i. e.,

$$
\xi_{1}=\xi\left(t_{1}\right)=x_{1}, \quad \eta_{1 i}=\eta_{i}\left(t_{1}\right)=y_{i}\left(x_{1}\right)=y_{1 i} .
$$

IV. The function $f$ does not vanish at the point of intersection of the curves $L$ and $E$; i. e., $f^{(1)}=f\left(x_{1} ; y_{1} ; y_{1}^{\prime}\right) \neq 0$.

We ask now what further conditions the extremal $E$ must satisfy in order to furnish a minimum for the integral (1) among all admissible curves which join a point on $L$ to $P_{2}$. For this purpose we compute the extremal integral along $E$. Since the coördinates of $P_{2}$ are fixed and the coördinates of $P_{1}$ are all functions of the single variable $t$, the extremal integral will become a function of $t$ alone,

$$
I\left(x_{1} ; y_{1} ; x_{2} ; y_{2}\right)=I\left(\xi(t) ; \eta(t) ; x_{2} ; y_{2}\right)=\Gamma(t),
$$

which must satisfy the ordinary conditions for a minimum at $t_{1}$, namely,

$$
\Gamma^{\prime}\left(t_{1}\right)=0, \quad \Gamma^{\prime \prime}\left(t_{1}\right) \geqq 0 .
$$

By the use of formulæ (12), we find:

$$
\Gamma^{\prime}\left(t_{1}\right)=\frac{\partial I}{\partial x_{1}} \xi^{\prime}+\left.\sum_{i} \frac{\partial I}{\partial y_{1 i}} \eta_{i}^{\prime}\right|_{1}=-\xi_{1}^{\prime} f^{(1)}+\sum_{i} f_{n+i}^{(1)}\left(\xi_{1}^{\prime} y_{1 i}^{\prime}-\eta_{1 i}^{\prime}\right)
$$

Its vanishing requires, as is well known, that $L$ must cut the extremal $E$ transversally at $P_{1}$. This condition of transversality, together with condition IV implies that

$$
\sum_{i} c_{i}^{2} \neq 0
$$

where $c_{i}=\xi_{1} y_{1 i}^{\prime}-\eta_{1 i}^{\prime}$.

A first value for $\Gamma^{\prime \prime}(t)$ is given by the formula

$$
\begin{aligned}
\Gamma^{\prime \prime}(t)=\frac{\partial I}{\partial x_{1}} \xi^{\prime \prime}+\sum_{i} \frac{\partial I}{\partial y_{1 i}} \eta_{i}^{\prime \prime}+\frac{\partial^{2} I}{\partial x_{1}^{2}} \xi^{2}+2 \sum_{i} \frac{\partial^{2} I}{\partial x_{1} \partial y_{1 i}} \xi^{\prime} \eta_{i}^{\prime} & \\
& +\sum_{i j} \frac{\partial^{2} I}{\partial y_{1 i} \partial y_{1 j}} \eta_{i}^{\prime} \eta_{j}^{\prime} .
\end{aligned}
$$

* Ibid., pp. 610-619. 
In the expressions (17) for the second derivatives of $I$ we replace the solutions $u_{k j}$ of the Jacobi equations by the fundamental system of solutions $\zeta_{r k}$ whose matrix reduces at $x=x_{1}$ to the identity matrix. Then it follows that

$$
u_{j k}=\zeta_{j k}-\sum_{r=n+1}^{2 n} \frac{Z^{(j k)}\left(x_{2}\right)}{Z\left(x_{2}\right)} \zeta_{r k}, \quad u_{j k}^{\prime}\left(x_{1}\right)=-\frac{Z^{(j k)}\left(x_{2}\right)}{Z\left(x_{2}\right)},
$$

where $Z(x)$ is the determinant $\left|\zeta_{r k}\right|(r=n+1, \cdots, 2 n ; k=1, \cdots, n)$, and $Z^{(j k)}(x)$ is this determinant after its $k$ th column has been replaced by $\zeta_{j 1}, \cdots, \zeta_{j n}$. With the help of $\left(17_{1}\right),\left(17_{4}\right)$, and $\left(17_{8}\right)$ equation (24) then takes the form

where

$$
\Gamma^{\prime \prime}\left(t_{1}\right)=A\left(x_{1}\right)+B\left(x_{1}, x_{2}\right)
$$

$$
\begin{gathered}
A\left(x_{1}\right)=-f^{(1)} \xi_{1}^{\prime \prime}-f_{0}^{(1)} \xi_{1}^{\prime 2}+\sum_{i} f_{n+i}^{(1)}\left(\xi_{1}^{\prime \prime} y_{1 i}^{\prime}-\eta_{1 i}^{\prime \prime}\right) \\
\quad+\sum_{i} \xi_{1}^{\prime} f_{i}^{(1)}\left(\xi_{1}^{\prime} y_{1 i}^{\prime}-2 \eta_{1 i}^{\prime}\right)-\sum_{i j} f_{n+i, j}^{(1)} c_{i} c_{j}, \\
B\left(x_{1}, x_{2}\right)=\frac{1}{Z\left(x_{2}\right)} \sum_{i j k} Z^{(j k)}\left(x_{2}\right) R_{i k}\left(x_{1}\right) c_{i} c_{j} .
\end{gathered}
$$

We investigate now the behavior of the function $B(x)=B\left(x_{1}, x\right)$ as $x$ varies from $x_{1}$ to $x_{1}^{\prime}$, merely sketching in the briefest way the proofs of the results. We find

$$
B^{\prime}(x)=-\frac{1}{R\left(x_{1}\right) Z^{2}} \sum_{i j} \beta_{i j} c_{i} c_{j}
$$

where $R(x)$ is the determinant $\left|R_{i k}\right|$ and

$$
\begin{aligned}
\beta_{i j}=-R\left(x_{1}\right) \sum_{k} R_{i k}\left(x_{1}\right)\left[Z Z^{(j k)^{\prime}}-Z^{\prime}\right. & \left.Z^{(j k)}\right] \\
& =\sum_{k l t s} R_{i k}\left(x_{1}\right) Z_{k l} \rho_{l s} Z_{t s} R_{t j}\left(x_{1}\right),
\end{aligned}
$$

in which $Z_{k l}$ is the cofactor of $\zeta_{k l}$ in $Z(x)$, and $\rho_{l s}$ the cofactor of $R_{l s}(x)$ in $R(x)$. The last expression for $\beta_{i j}$ is derived from the first by applying successively formulæ (70), (65), and (55) in Chapter XII of Bolza's Vorlesungen, and remembering that the function $\psi$ of formula (55) is a constant which can be evaluated at $x_{1}$, where the values of the functions $\zeta_{r k}$ are prescribed. In these formulæ, the solutions $z, u$ of Jacobi's equations are to be replaced by solutions of the matrix $\zeta_{r k}$ defined above; and for our case the multipliers $\rho, r$ are all equal to zero identically, since there are no adjoined differential equations.

The quadratic form $\sum \beta_{i j} c_{i} c_{j}$ is positive, on account of condition (23) together with the fact that it is derived from the quadratic form $\sum \rho_{l s} x_{l} x_{s}$ by two linear transformations whose determinants are equal to $Z$ and $R$ respectively. We have $Z \neq 0$ because the Mayer condition is fulfilled and 
because the Mayer determinant (14) reduces to $Z$ when the functions (5) are expressed linearly in terms of the functions $\zeta_{r k}$. Further $R \neq 0$ on account of condition I, and since the matrix $\left|\rho_{l_{s}}\right|$ is the matrix of the cofactors of $R$, the form $\sum \rho_{l s} x_{l} x_{s}$ is positive. If we now apply condition $I$ once more, we conclude that $B^{\prime}(x)$ is steadily negative on the interval $x_{1}<x<x_{1}^{\prime}$.

The initial values of the functions $\zeta_{r k}$ at $x=x_{1}$ are known, and hence the first few terms of the Taylor expansions for the numerator and denominator of $B(x)$ can be found. It is found that in the neighborhood of $x_{1}$, we may write

$$
B(x)=\frac{1}{x-x_{1}}\left\{\sum_{i j} R_{i j}\left(x_{1}\right) c_{i} c_{j}+a_{1}\left(x-x_{1}\right)+\cdots\right\},
$$

so that $B(x)$ approaches $+\infty$ as $x$ approaches $x_{1}$ from the right.

Finally, setting $x$ equal to $x_{1}^{\prime}$ in the two expressions for $\beta_{i j}$ given in formula (25), we obtain, since $Z\left(x_{1}^{\prime}\right)=0$,

$$
R\left(x_{1}\right) \sum_{k} R_{i k}\left(x_{1}\right) Z^{\prime} Z^{(j k)}=\sum_{k l t s} R_{i k}\left(x_{1}\right) Z_{k l} \rho_{l s} Z_{t s} R_{t j}\left(x_{1}\right),
$$

the argument $x$ being everywhere replaced by $x_{1}^{\prime}$. It follows from this that the numerator of $B\left(x_{1}^{\prime}\right)$ reduces to

$$
\frac{1}{R\left(x_{1}\right) Z^{\prime}} \sum_{i j} \beta_{i j} c_{i} c_{j}
$$

which is different from zero; further, since the denominator vanishes, $B(x)$ becomes infinite as $x$ approaches $x_{1}^{\prime}$, and it must approach $-\infty$, because $B^{\prime}(x)$ is negative.

From these facts it follows that the function $B\left(x_{1}, x\right)$ decreases univariantly from $+\infty$ to $-\infty$ as $x$ increases from $x_{1}$ to $x_{1}^{\prime}$. Hence there is an unique value $x_{1}^{\prime \prime}$ between $x_{1}$ and $x_{1}^{\prime}$ such that $\Gamma^{\prime \prime}\left(t_{1}\right)$ is positive for every value $x_{2}$ satisfying the condition $x_{1}<x_{2}<x_{1}^{\prime \prime}$. The point defined on $E$ by $x_{1}^{\prime \prime}$ is called the "focal point" of $L$ on $E$. We have therefore the following theorem:

Theorem II. If an extremal arc $E$ with the properties I-IV minimizes the integral (1) with respect to arcs joining a fixed curve $L$ to a fixed point $P_{2}$, then it must be cut transversally by $L$ at their point of intersection $P_{1}$, and it must further be such that the focal point of $L$ on $E$ is not between $P_{1}$ and $P_{2}$.

The UnIversity of Wisconsin 